\title{
Alterations in bone turnover by isoflavone aglycone supplementation in relation to estrogen receptor $\alpha$ polymorphism
}

\author{
HIRONOBU KATSUYAMA ${ }^{1}$, MASAYUKI ARII ${ }^{5}$, HAJIME HINENOYA ${ }^{1}$, MASAHIRO MATSUSHIMA ${ }^{1}$, \\ SHIGEKO FUSHIMI ${ }^{1}$, MASAFUMI TOMITA ${ }^{2}$, TOSHIKO OKUYAMA ${ }^{2}$, KAZUO HIDAKA ${ }^{3}$, \\ YOKO WATANABE $^{3}$, YOSHIE TAMECHIKA ${ }^{4}$ and KIYOFUMI SAIJOH ${ }^{6}$
}

\begin{abstract}
Departments of ${ }^{1}$ Public Health, ${ }^{2}$ Medical Toxicology, ${ }^{3}$ Biochemistry, and ${ }^{4}$ Clinical Laboratory, Kawasaki Medical School, Kurashiki, Okayama 701-0192; ${ }^{5}$ Research and Development Division, Kikkoman Corporation, Tokyo 105-8428;

${ }^{6}$ Department of Hygiene, Kanazawa University School of Medicine, Kanazawa, Ishikawa 920-8640, Japan
\end{abstract}

Received January 26, 2010; Accepted March 22, 2010

DOI: $10.3892 / \mathrm{mmr} 00000293$

\begin{abstract}
Soybean isoflavones have structural similarity to estrogen and have attracted much attention due to their prevention of postmenopausal symptoms. It is critical for women to maintain a high bone mineral density (BMD) prior to menopause to prevent osteoporosis. In the present study, the effect of isoflavone aglycone (IA) supplementation on bone turnover was examined in relation to the estrogen receptor $\alpha(E R \alpha)$ polymorphism. Natural isoflavones are glycosides that must be hydrolyzed to aglycones by intestinal microflora to have an effect. To avoid interference by flora, IA (30 mg/day) (but not isoflavones) or a placebo were administered as a supplement for 3 months to a Japanese population consisting of 81 premenopausal women. Due to variations in the intestinal flora, some but not all subjects were able to further metabolize IA into equol. Differences between equol producers and non-producers were also considered. To estimate BMD, the osteo-sono-assessment index (OSI) was determined by measuring bone density at the calcaneus and levels of bone biochemical markers (bonespecific alkaline phosphatase, $\alpha$-carboxylated osteocalcin, undercarboxylated osteocalcin and deoxypridinoline) before and after supplementation. DNA samples from the subjects were examined for the presence of the $X b a \mathrm{I}$ restriction fragment length polymorphism (RFLP) in intron 1. According to univariate analysis, IA had a favorable effect on the OSI of subjects with the $\mathrm{X}$ allele, with $\mathrm{X}$ designated RFLP undigested by $X b a \mathrm{I}$, although the difference was not statistically significant. Alterations in the levels of bone biochemical markers were also
\end{abstract}

Correspondence to: Dr Hironobu Katsuyama, Department of Public Health, Kawasaki Medical School, 577 Matsushima, Kurashiki, Okayama 701-0192, Japan

E-mail:katsu@med.kawasaki-m.ac.jp

Key words: isoflavone aglycone, estrogen receptor $\alpha$ polymorphism, biochemical markers of bone turnover, osteosono-assessment index not significant. Thus, a further logistic regression analysis was performed. This indicated that subjects with the XX homozygote administered the IA supplement were less likely to have reduced OSI values. Although equol has been proposed to have the highest phytoestrogen activity, its effect was not apparent. Thus, low-dose IA supplementation is useful for maintaining BMD in premenopausal XX subjects, independent of equol.

\section{Introduction}

Bone mineral density (BMD), one of the most significant predictors of osteoporotic fracture, is influenced by both environmental and genetic factors (1). Although osteoporosis results in inadequate skeletal strength, predisposing its sufferers to fracture, most osteoporotic patients are diagnosed after the occurrence of fractures since osteoporosis itself lacks specific symptoms. Even if osteoporosis is diagnosed before a fracture occurs, it is difficult to recover BMD in a short period of time. In females, BMD reaches its maximum in adolescence and gradually decreases until menopause, after which it decreases dramatically (2). Since a reduction in BMD due to menopause is unavoidable, it is critical for females to achieve a maximum level during adolescence and to prevent a reduction in BMD before menopause.

Soy is part of the traditional diet of Asians, and epidemiological data indicate that Asian populations have a lower rate of osteoporotic fractures, cardiovascular diseases, postmenopausal symptoms, and certain types of cancers than Western populations (3). Soybean isoflavones have a structural similarity to estrogen and act as agonists of estrogen receptors; thus, they are called phytoestrogens. However, isoflavones exist as naturally occurring glycosidic forms (4) and need to be hydrolyzed by intestinal flora to isoflavone aglycones (IA), mainly consisting of genistein and daidzein (5). Numerous studies suggest that daidzein is further metabolized into equol by Bifidobacterium, Escherichia coli, Bacteroides ovatus, Ruminococcus productus and Streptococcus intermedius $(6,7)$. However, the main bacteria responsible for daidzein degradation have not been definitively identified (8), and in 
humans only $30-50 \%$ of individuals are capable of producing equol from daidzein (9). IA and equol are structurally similar to $17 \beta$-estradiol and have an affinity to estrogen receptors, thus possessing estrogenic activity. Dietary equol exerts mild estrogenic effects in the pituitary, uterus, mammary glands, liver and fat tissue, but shows limited bone-sparing effects in ovariectomized rats (10).

Estrogen deficiency plays a major role in the pathogenesis of postmenopausal osteoporosis. Estrogen deficiency is characterized by increased bone resorption and a remodeling imbalance (11). Richard et al (12) found that genistein increases the gene expression of the progesterone receptor, proteoglycan versican and alkaline phosphatase, but inhibits osteopontin gene expression and interleukin- 6 production in human fetal osteoblast cells expressing estrogen receptor $\alpha$ (ER $\alpha)$. Three polymorphisms have been reported in the ER $\alpha$ gene, $X b a \mathrm{I}$ restriction fragment length polymorphism (RFLP) in intron I, $P v u I I$ RFLP in the same intron, and TA-variable number of tandem repeat (TA-VNTR) in the promoter region (13). The Px haplotype, in which $\mathrm{P}$ lacks a $P v u I I$ site and $\mathrm{x}$ possesses an $X b a I$ site, was found to be significantly associated with low BMD (14). Recent meta-analysis data suggest that the XX of the ER $\alpha$ polymorphism may be related to high BMD and low fracture risk, while $P v u I I$ and TA-VNTR show no relationship to the risk of fracture $(13,15) . X b a$ I RFLP therefore appears to be important for the maintenance of BMD. However, little is known about the role of this polymorphism in regards to the effect of nutritional IA on BMD.

In the present study, IA (30 mg/day) was administered as a supplement to premenopausal women employed at local hospitals in Japan for 3 months, and alterations in the levels of bone biochemical markers were examined in relation to the $X b a \mathrm{I}$ RFLP of ER $\alpha$.

\section{Subjects and methods}

Subjects. A total of 98 premenopausal women employed at local hospitals in Japan were recruited. All subjects gave their informed consent for participation in the study. The women were divided into two groups and administered an IA supplement (30 mg/day) or a placebo for 3 months. Seventeen women withdrew from the study, as IA triggered a new menstrual cycle or exaggerated menstrual symptoms.

This study was approved by the Medical Ethics Committee of Kawasaki Medical School and Kawasaki Medical School Hospital (no. 100).

Bone mineral density measurement. BMD was measured by an ultrasound method to obviate X-ray exposure. The speed of sound and transmission index at the calcaneus were measured, and the osteosono-assessment index (OSI) was calculated by the quantitative ultrasound method (AOS-100, Aloka Co., Ltd., Tokyo). OSI was used as the index of BMD.

Biochemical markers of bone turnover. Second-morning urine specimens were collected and blood samples were obtained at the time of QUS measurement. Both serum and urine specimens were stored at $-20^{\circ} \mathrm{C}$ until measurement. As a bone formation marker, serum $\alpha$-carboxylated osteocalcin (Gla) was measured using the Gla EIA kit (Takara, Osaka,

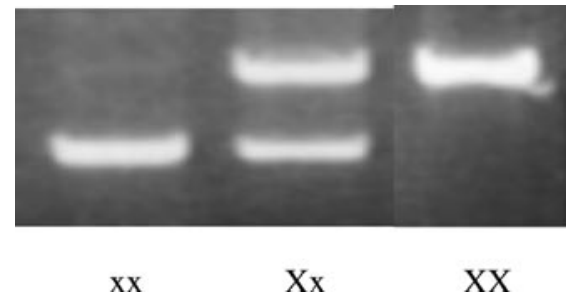

Figure 1. Representative RFLP of the estrogen receptor $\alpha(\mathrm{ER} \alpha)$ gene. $X b a \mathrm{I}$ was capable of digesting PCR-amplified intron I of the $\mathrm{x}$ allele, but not of the $\mathrm{X}$ allele.

Japan). Osteocalcin correlates well with bone formation, but is readily subject to rapid degradation in serum (16). Not only Gla and undercarboxylated osteocalcin (uc-OC), but also fragments of these in various sizes, coexist in the circulation, therefore different immunoassays yield varying results (17). In the present study, a kit utilizing an antibody specific to $17 \mathrm{Gla}$ and its adjacent region was used to isolate Gla. Although $\alpha$-carboxylation preferentially occurs at residues 21 and 24 (18), 17Gla is essential for a Ca-dependent conformational transition (19), indicating that the 17Gla-specific antibody recognizes fully active Gla. To measure uc-OC as a resorption marker, a kit using a uc-OC-specific antibody was utilized. In addition, another formation marker, serum bone-specific alkaline phosphatase (BAP), and another resorption marker, deoxypyridinoline (DPD), were measured in urine by ELISA. DPD values were corrected by creatinine in urine. The menstrual cycle of each subject was not taken into account, as the effects of the menstrual cycle on bone turnover are thought to be minimal (20). These biochemical markers were assessed as percent changes after supplementation.

Measurement of equol. Serum equol, a metabolite of daidzein, was measured by high performance liquid chromatography according to the method of Hidaka et al (21). An equol producer was defined as a subject with a serum concentration of equol $\geq 10 \mathrm{ng} / \mathrm{ml}$.

XbaI RFLP. Blood samples were obtained at the time of examination, and genomic DNA was extracted from leukocytes. Intron I of the ER $\alpha$ gene, where the $\mathrm{XbaI}$ digestion site is located, was amplified by polymerase chain reaction (PCR) using a set of primers [forward: CTGCCACCCTATCTGTAT CTTTTCCTATTCTCC and reverse: TCTTTCTCTGCCACC CTGGCGTCGATTATCTGA (22)]. The obtained products were digested with $\mathrm{XbaI}$. The genotype was designated as $\mathrm{X}$ or as $\mathrm{X}$ when the product was digested or not digested, respectively. Fig. 1 shows the representative results distinguishing the genotypes $\mathrm{xx}, \mathrm{xX}$ and $\mathrm{XX}$.

Statistical analysis. The Student's t-test was performed to compare differences in OSI and biochemical markers of bone turnover for each group. Friedman analysis was performed and box and whisker plots were constructed to examine the interaction between XbaI RFLP and IA supplementation. Odds ratios as estimated risk with $95 \%$ confidence intervals were calculated by logistic regression analysis using JMP 7.0 (SAS Corp.). 
Table I. Baseline characteristics of the subjects.

\begin{tabular}{lcc}
\hline & IA group & Placebo group \\
\hline No. of subjects & 40 & 41 \\
Mean age (years) & $35.5 \pm 8.9$ & $36.0 \pm 8.4$ \\
Equol producers & 19 & 14 \\
ER & & \\
XX & 8 & 12 \\
Xx & 15 & 18 \\
XX & 17 & 11 \\
OSI & $2.67 \pm 0.24$ & $2.63 \pm 0.23$ \\
BAP (U/l) & $21.4 \pm 8.6$ & $22.0 \pm 6.1$ \\
DPD (nM/mM Cr) & $6.67 \pm 2.09$ & $6.10 \pm 1.90$ \\
Gla (ng/ml) & $4.76 \pm 2.45$ & $4.56 \pm 1.89$ \\
uc-OC (ng/ml) & $2.08 \pm 1.14$ & $1.67 \pm 0.93$ \\
\hline
\end{tabular}

No statistical differences were observed between each group (t-test). IA, isoflavone aglycone. OSI, osteo-sono-assessment index; BAP, bone-specific alkaline phosphatase; DPD, deoxypridinoline; Gla, $\alpha$-carboxylated osteocalcin; uc-OC, undercarboxylated osteocalcin.

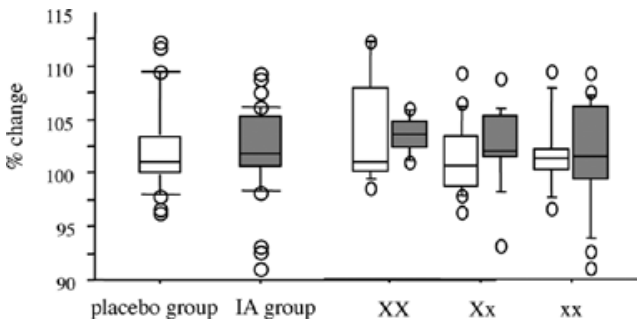

Figure 2. Box and whisker plots of percent changes in OSI 3 months after IA supplementation in the IA and placebo groups as well as in each genotype group. OSI showed no statistical differences among the groups (Friedman analysis). The top section of each box indicates the third quartile and the bottom indicates the first quartile. Horizontal and vertical bars (whiskers) in the box indicate the midpoint of distribution and allowable observation, respectively. Open circles indicate the outling values.

\section{Results}

Baseline characteristics of the subjects. Since subjects were randomly allocated to each group, we first confirmed that there were no significant differences in basic characteristics, including mean age, the number of equol producers and the number of subjects with XbaI RFLP, between the IA and placebo groups (Table I). No statistical difference in OSI was observed. The levels of bone formation markers, BAP and Gla, and those of bone resorption markers, DPD and uc-OC, were not significantly different between each group. Differences in the intake of soybean and other nutrients were not statistically significant among the subjects (data not shown).

Changes in bone biochemical markers. Percent changes in bone biochemical markers from the baseline 3 months after supplementation were compared among the groups. Although no statistical differences were observed, the IA group showed a higher \%OSI than the placebo group, due mainly to the
Table II. Risk determinants for a reduction in osteo-sonoassessment index (OSI) values.

\begin{tabular}{lcc}
\hline & Odds ratio & 95\% CI \\
\hline IA group (0, placebo; 1, IA) & 0.32 & $0.11-0.85$ \\
Equol (0, non producer; 1, producer) & 1.04 & $0.38-2.86$ \\
XbaI RFLP (1, XX; 2, Xx; 3, xx) & 1.90 & $1.01-3.76$ \\
\%BAP & 0.98 & $0.96-1.00$ \\
\%DPD & 1.00 & $0.98-1.02$ \\
\%Gla & 1.00 & $0.99-1.01$ \\
\%uc-OC & 1.00 & $0.99-1.00$ \\
\hline
\end{tabular}

Odds ratios for the reduction in OSI after supplementation were calculated according to potential risk factors. CI, confidence interval; IA, isoflavone aglycone; BAP, bone-specific alkaline phosphatase; DPD, deoxypridinoline; Gla, $\alpha$-carboxylated osteocalcin; uc-OC, undercarboxylated osteocalcin.
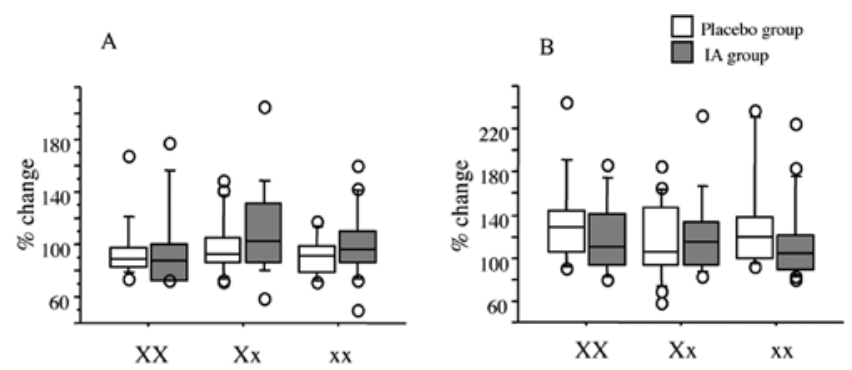

C
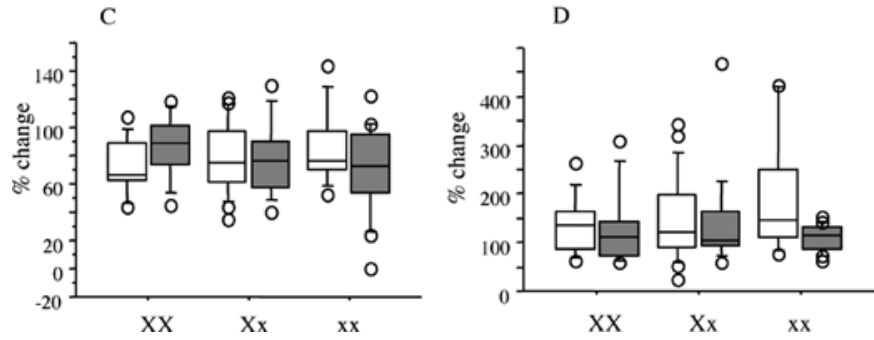

Figure 3. Box and whisker plots of percent changes in bone biochemical markers 3 months after IA supplementation in each genotype group. The BAP (A), Gla (B), DPD (C) and uc-OC (D) bone resorption markers showed no statistical differences among the groups (Friedman analysis). Symbols are as in Fig. 2.

effect of IA on the ER $\alpha$ X allele group (Fig. 2). No marked differences among the groups were observed regarding changes in bone formation markers \%BAP and \% Gla (Fig. 3A and $\mathrm{B}$ ), nor were changes in bone resorption markers \% DPD and \%uc-OC noted (Fig. 3C and D). Since univariate analyses were insufficient for clarifying the effect of IA supplementation, logistic regression analysis was further performed as a multivariate analysis.

Risk determinants for the reduction in OSI values using logistic regression analysis. In the logistic regression analysis, odds ratios for the reduction in OSI values were calculated according to potential risk factors (Table II). IA supplementa- 
Table III. Risk determinants for the decrease in osteosono-assessment index (OSI) for each XbaI RFLP.

\begin{tabular}{lcccccc}
\hline & \multicolumn{2}{c}{$\mathrm{XX}$} & \multicolumn{2}{c}{$\mathrm{Xx}$} & \multicolumn{2}{c}{$\mathrm{xx}$} \\
\hline IA group (0, placebo; 1, IA) & 0.01 & $0.00-0.41$ & 0.26 & $0.04-1.29$ & 1.99 & $0.20-23.76$ \\
Equol (0, non producer; 1, producer) & 2.04 & $0.05-152.95$ & 0.31 & $0.04-1.87$ & 0.49 & $0.05-3.64$ \\
\%BAP & 0.92 & $0.69-1.02$ & 0.99 & $0.96-1.02$ & 0.96 & $0.91-1.01$ \\
\%DPD & 1.04 & $0.95-1.24$ & 1.00 & $0.96-1.05$ & 1.00 & $0.97-1.04$ \\
\%Gla & 0.98 & $0.01-1.03$ & 0.98 & $0.94-1.01$ & 1.01 & $099-1.04$ \\
\%uc-OC & 1.01 & $0.99-1.04$ & 0.99 & $0.98-1.01$ & 1.00 & $0.99-1.01$ \\
\hline
\end{tabular}

For the calculation of the Odds ratio, refer to Table II legend. IA, isoflavone aglycone; BAP, bone-specific alkaline phosphatase; DPD, deoxypridinoline; Gla, $\alpha$-carboxylated osteocalcin; uc-OC, undercarboxylated osteocalcin.

tion significantly reduced the risk. XbaI RFLP was found to be a risk, but whether or not allele $\mathrm{x}$ itself or the $\mathrm{xx}$ homozygote was responsible for this risk could not be determined by the analysis. In order to clarify the effect of XbaI RFLP, logistic regression analyses were further performed for each genotype.

A favorable effect of IA was statistically significant only in subjects with $\mathrm{XX}$, indicating that the $\mathrm{x}$ allele itself was the risk (Table III). The effect on the alteration of OSI was independent of equol production. None of the formation markers and resorption markers altered OSI.

\section{Discussion}

Postmenopausal female subjects who were administered $37.3 \mathrm{mg} /$ day isoflavone for 10 weeks were found to have a lower urinary DPD level than the control group (23), and those who were treated with $80 \mathrm{mg}$ isoflavone for 1 year showed higher bone mineral contents in the total hip and trochanter (24). Subjects who received a supplement of $110 \mathrm{mg} /$ day IA for 6 months demonstrated an effective decrease in bone resorption (25). Thus, IA not only decreases bone resorption markers, but also protects against actual BMD loss. The present study aimed to ascertain whether IA was useful for the maintenance of BMD in premenopausal women. Isoflavone intake is limited to $30 \mathrm{mg}$ /day by the Food Safety Commission, Japan (26); therefore, in the present study, we administered a dose as low as $30 \mathrm{mg}$ /day of IA alone. The fact that IA supplementation in premenopausal subjects failed to show apparent effects as determined by univariate analyses alone can be attributed to this low administered dose. Nonetheless, $20.5 \%$ of subjects in the IA group complained of some symptoms even at this low dose during the short supplementation period. The existence of such symptoms confirms that IA is potent even in premenopausal women.

In the abovementioned research involving postmenopausal women, the relationship between isoflavone intake and estrogen receptor polymorphisms was not taken into account. Our results indicate that subjects with $\mathrm{ER} \alpha \mathrm{XX}$ were more sensitive to the beneficial effects of IA supplementation. Additionally, even before menopause, IA supplementation seemed to be useful for the maintenance of OSI. On the other hand, the effect of IA supplementation did not differ between equol producers and non-producers. The half-life of equol in the body is significantly longer than that of daidzein or genistein, and equol is reported to be present at high levels in the blood of equol producers (27). Equol is considered to be the most biologically active phytoestrogen, followed by genistein and daidzein. Equol is capable of improving fracture healing in ovariectomy-induced osoteoporotic bones in mice (28). The lack of an apparent effect of equol in the present study was probably due to the fact that the low dose of IA was already sufficient to confer a beneficial effect on subjects with XX, or that IA had beneficial effects on BMD besides its action as a phytoestrogen. Hence, further study is necessary in order to clarify the pharmacological difference between equol and IA itself.

In conclusion, IA supplementation was effective for reducing the risk of OSI reduction in premenopausal subjects with the XX homozygote. It seems necessary to explore whether or not the $\mathrm{X}$ haplotype itself confers a beneficial effect. Moreover, the importance of this polymorphism should be considered in postmenopausal research.

\section{Acknowledgements}

We thank Tsutomu Higashimura, chief radiologist, and Noriko Maeda, administrative dietitian, of Kosei Hospital, for their technical support. This study was supported, in part, by a Research Project Grant (no. 17-501) from Kawasaki Medical School.

\section{References}

1. Eisman JA: Genetics of osteoporosis. Endocr Rev 20: 788-804, 1999.

2. Yamazaki K, Kushida K, Ohkuma A, Sano M and Inoue T: Ultrasound bone densitometry of the os calcis in Japanese women. Osteoporos Int 4: 220-225, 1994.

3. Adlercreutz $\mathrm{H}$ and Mazur W: Phyto-oestrogens and Western diseases. Ann Med 29: 95-120, 1997.

4. Nielsen ILF and Williamson G: Review of the factors affecting bioavailability of soy isoflavones in humans. Nutr Cancer 57: 1-10, 2007.

5. Yamaguchi M: Regulatory mechanism of food factors in bone metabolism and prevention of osteoporosis. Yakugaku Zasshi 126: 1117-1137 2006.

6. Tsangalis D, Wilcox G, Shah NP and Stojanovska L: Bioavailability of isflavone phytoestrogens in postmenopausal women consuming soya milk fermented with probiotic bifidobacteria. Br J Nutr 93: 867-877, 2005.

7. Hur HG, Lay JO Jr, Beger RD, Freeman JP and Rafii F: Isolation of human intestinal bacteria metabolizing the natural isoflavone glycosides daidzin and genistin. Arch Microbiol 174: 422-428, 2000. 
8. Atkinson C, Frankenfeld CL and Lampe JW: Gut bacterial metabolism of the soy isoflavone daidzein: exploring the relevance to human health. Exp Biol Med 230: 155-170, 2005.

9. Setchell KD: Absorption and metabolism of soy isoflavones from food to dietary supplements and adults to infants. J Nutr 130: 654s-655s, 2000.

10. Rachon D, Seidlova-Wuttke D, Vortherms T and Wuttke W: Effects of dietary equol administration on ovariectomy induced bone loss in Sprague-Dawley rats. Maturitas 58: 308-315, 2007.

11. Turner RT, Riggs BL and Spelsberg TC: Skeletal effects of estrogen. Endocr Rev 15: 275-300, 1994.

12. Richard DJ, Monroe DG, Ruesink TJ, Khosla S, Riggs BL and Spelsberg TC: Phytoestrogen genistein acts as an estrogen agonist on human osteoblastic cells through estrogen receptors $\alpha$ and $\beta$. J Cell Biochem 89: 633-646, 2003.

13. Ioannidis JP, Stavrou I, Trikalinos TA, et al: Association of polymorphisms of the estrogen receptor $\alpha$ gene with bone mineral density and fracture risk in women: a meta-analysis. J Bone Miner Res 17: 2048-2060, 2002

14. Kobayashi S, Inoue S, Hosoi T, Ouchi Y, Shiraki M and Orimo H Association of bone mineral density with polymorphism of the estrogen receptor gene. J Bone Miner Res 11: 306-311, 1996.

15. Ioannidis JP, Ralston SH, Bennett ST, et al: Differential genetic effects of ESR1 gene polymorphisms on osteoporosis outcomes. JAMA 292: 2105-2114, 2004.

16. Garnero P, Grimaux M, Seguin P and Delmas PD: Characterization of immunoreactive forms of human osteocalcin generated in vivo and in vitro. J Bone Miner Res 9: 255-264, 1994.

17. Seibel MJ and Woitge HW: Basic principle and clinical applications of biochemical markers of bone metabolism. J Clin Densitometry 2: 299-321, 1999.

18. Benton ME, Price PA and Suttie JW: Multi-site-specificity of the vitamin K-dependent carboxylase: in vitro carboxylation of des- $\alpha$-carboxylated bone Gla protein and des- $\alpha$-carboxylated pro bone Gla protein. Biochemistry 34: 9541-9551, 1995.

19. Nakao M, Nishiuchi Y, Nakata M, Kimura T and Sakakibara S Synthesis of human osteocalcins: $\alpha$-carboxyglutamic acid at position 17 is essential for a calcium-dependent conformational transition. Pept Res 7: 171-174, 1994.
20. Hannon R and Eastell R: Preanalytical variability of biochemical markers of bone turnover. Osteoporos Int 6: s30-s44, 2000

21. Hidaka K, Higashi M, Watanabe Y, Tomita M, Katsuyama H and Tamechika Y: An improved analytical method for detection of isoflavones in plasma by high performance liquid chromatography. Kawasaki Ikaishi Arts Sci 33: 9-15, 2007.

22. Strand M, Soderstrom I, Wiklund PG, Hallmans G, Weinehall L, Soderberg S and Olsson T: Estrogen receptor alpha gene polymorphisms and first-ever intracerebral hemorrhage. Cerebrovasc Dis 24: 500-508, 2007.

23. Yamori Y, Moriguchi EH, Teramoto T, et al: Soybean isoflavones reduce postmenopausal bone resorption in female Japanese immigrants in Brazil: A ten-week study. J Am Coll Nutr 21: 560-563, 2002.

24. Chen Y, Ho SC, Lam SSH, Ho SSS and Woo JLF: Soy isoflavones have a favorable effect on bone loss in Chinese postmenopausal women with lower bone mass: a double-blind, randomized, controlled trial. J Clin Endocrinol Metab 88: 4740-4747, 2003.

25. Harkness LS, Fielder K, Sehgal AR, Oravec D and Lerner E: Decreased bone resorption with soy isoflavone supplementation in postmenopausal women. J Womens Health 13: 1000-1007, 2004.

26. Novel Foods Expert Committee: Fundamental concepts in the safety assessment of foods containing soy isoflavones for the purpose of specified health use. Food Safety Commission, Tokyo, Japan, p45. 2006.

27. Kang NJ, Lee KW, Rogozin EA, Cho YY, Heo YS, Bode AM, Lee HJ and Dong Z: Equol, a metabolite of the soybean isoflavone daidzein, inhibits neoplastic cell transformation by targeting the MEK/ERK/p90RSK/activator protein-1 pathway. J Biol Chem 282: 32856-32866, 2007.

28. Kolios L, Sehmisch S, Daub F, Rack T, Tezval M, Stuermer KM and Stuermer EK: Equol, but not genistein improves early metaphyseal fracture healing in osteoporotic rats. Planta Med 75: $459-465,2009$ 